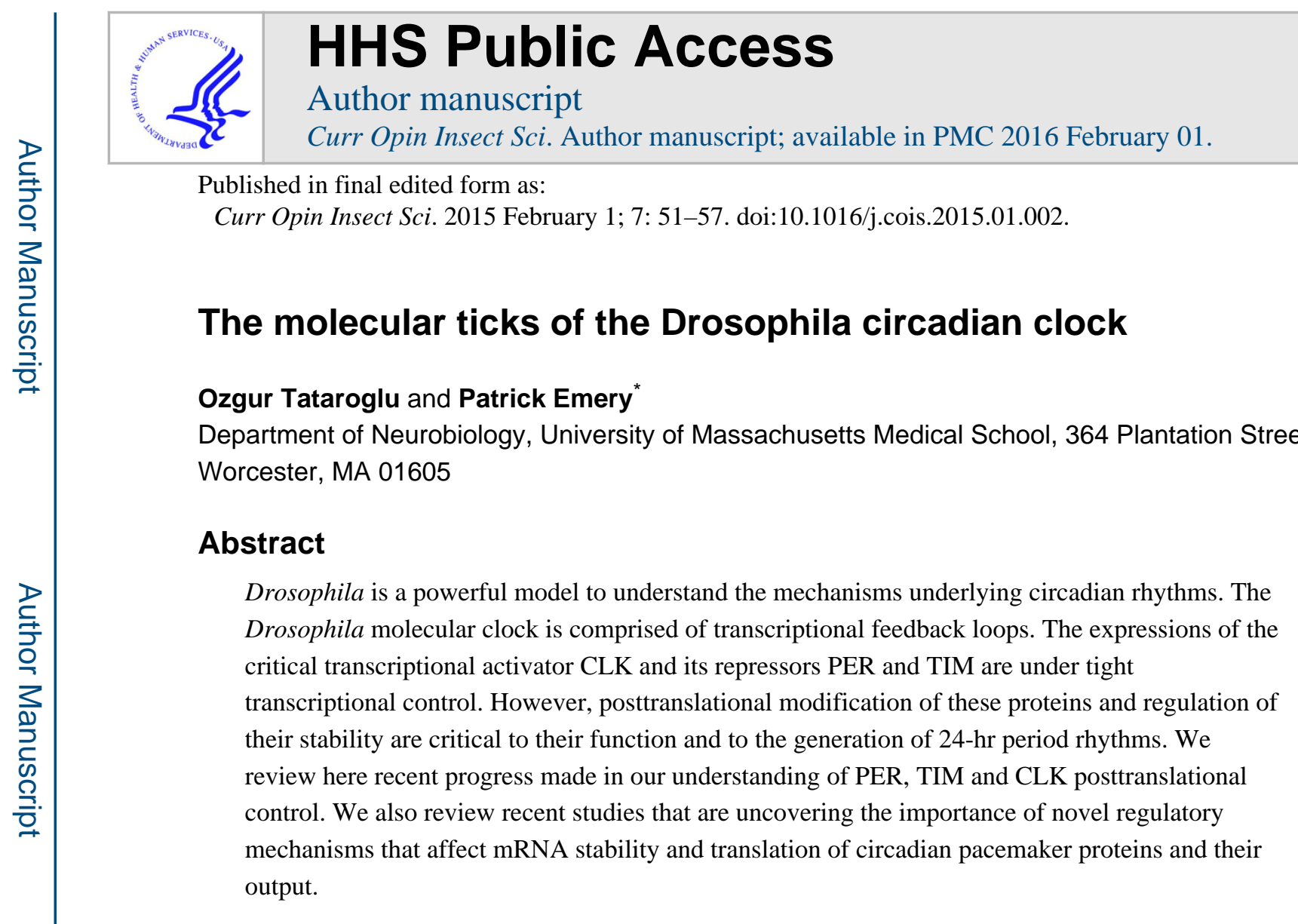

\title{
Introduction
}

Circadian clocks are molecular oscillators that enable organisms from cyanobacteria to humans to anticipate daily events that occur in their environment. Although circadian rhythms are self-sustained, they receive input from the environment such as light and temperature, which determine their phase. However, their period is independent of ambient temperature. Circadian clocks keep time throughout the day and seasons and thus have a broad impact on the biology of most organisms. They control behavioral, physiological and molecular rhythms such as locomotor activity, feeding, sleep, reproduction and gene expression [1]. They also have a key role in sun-compass navigation, allowing migratory animals such as monarch butterflies to compensate for time-of-day when using the sun for direction [2].

Historically, Drosophila has been at the forefront of circadian rhythm research due to its ease of use and its advanced molecular and genetic toolkit [3]. Importantly, the molecular circadian clock is highly conserved from Drosophila to humans and consists of interlocked transcriptional negative feedback loops (Figure 1). At the center of these loops are the transcription factors CLOCK (CLK) and CYCLE (CYC), which form a heterodimer that drives the expression of PERIOD (PER) and TIMELESS (TIM). PER and TIM function as a

\footnotetext{
*Corresponding author, Patrick.Emery@ umassmed.edu Phone: 508-856-6599.

Publisher's Disclaimer: This is a PDF file of an unedited manuscript that has been accepted for publication. As a service to our customers we are providing this early version of the manuscript. The manuscript will undergo copyediting, typesetting, and review of the resulting proof before it is published in its final citable form. Please note that during the production process errors may be discovered which could affect the content, and all legal disclaimers that apply to the journal pertain.
} 
heterodimeric repressor of CLK/CYC in the nucleus. In a second transcriptional feedback loop, CLK and CYC drive the expression of VRILLE (VRI) and PAR DOMAIN

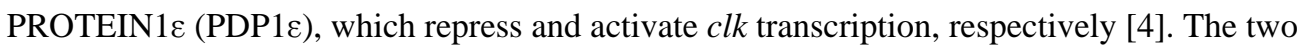
transcriptional feedback loops control multiple clock controlled genes (ccgs), but interestingly generate anti-phase mRNA oscillations [5]. PER and TIM synthesis and degradation is tightly regulated throughout the day, and this complex regulation is critical to set the pace of the circadian pacemaker. Indeed, delays in PER and TIM accumulation and nuclear translocation are thought to be critical for generating stable 24-hr period rhythms. PER and TIM abundances are tightly regulated at the transcriptional, post-transcriptional, and post-translational levels. Important progress has recently been made on the two latter regulatory levels, which will be the focus of this review.

\section{Post-Translational control}

\section{PER and TIM phosphorylation and ubiquitination}

Phosphorylation has been shown to have a critical role in determining the period of the clock. In fact, PER and TIM are phosphorylated at many sites throughout the day by several kinases. These include DOUBLETIME (DBT), SHAGGY (SGG), CASEIN KINASE2 (CK2) and NEMO (NMO) which regulate PER and TIM stability and nuclear entry, depending on the location and cooperativity of the target site for phosphorylation $[1,6]$ (Figure 1). For example, mutation of T610 on PER to alanine lengthens the period by only 2-3 hr while S613 mutation has minimal effect. However, simultaneous mutations of both sites to alanine results in reduced phosphorylation and increased stability of PER and a $\sim 30$ hr period length, suggesting that these sites cooperate to determine circadian period [7].

DBT - the Drosophila homolog of CASEIN KINASE1 $\delta / \varepsilon(C K 1 \delta / \varepsilon)$ - extensively phosphorylates PER throughout the day, which ultimately results in its degradation via the proteasome [5]. This is mediated by the F-box protein SLIMB binding to PER (Figure 1). This interaction is promoted by phosphorylation of PER's S47 [8-10]. Furthermore, this binding can be negatively regulated by NEMO and DBT-mediated phosphorylation at S585, S589 and S596. This inhibits DBT-mediated phosphorylation of S47 and thereby delaying PER degradation by SLIMB [11]. This shows that phosphorylation does not need to be in close proximity and distant sites can influence each other in regulating PER stability and function. Phosphorylations also occur on TIM and are mediated by kinases CK2 $a$ and SGG, which regulates TIM stability, similar to PER phosphorylation [12-14]. These studies show that phosphorylation events determine the speed of the clock by regulating the rate of nuclear accumulation of PER and TIM [13,14].

Interestingly, SLIMB also controls TIM stability, with the help of another ubiquitin (UB)ligase that contains CULLIN-3 (CUL3) [15••] (Figure 1). CUL3 preferentially complexes with hypo-phosphorylated TIM unbound to PER, while SLMB targets preferentially hyperphosphorylated TIM bound to PER. Disrupting CUL-3 function through RNAi or expression of a dominant-negative mutant leads to long period phenotypes and arrhythmicity. CUL-3 downregulation results in low amplitude TIM cycling, with elevated hypo-phosphorylated TIM during the subjective day. This would fit with a simple model in which CUL-3 preferentially degrades hypo-phosphorylated TIM (Figure 1). Unexpectedly however, the 
abundance of the hyper-phosphorylated TIM isoform is increased with the overexpression of a dominant-negative CUL-3 mutant. CUL-3 might thus also repress TIM phosphorylation, or destabilize this isoform. These phenotypic differences could reflect different efficiency of RNAi or overexpression of a dominant negative mutant to disrupt CUL-3 activity. Further studies are clearly needed to elucidate fully the role of CUL-3 in the circadian pacemaker

Formation of the PER/TIM heterodimer stabilizes PER, protecting it from phosphorylation and SLIMB degradation [8]. Also, PER and TIM phosphorylation is counteracted by the activity of protein phosphatases 2A (PP2A) on PER and 1A (PP1) on PER and TIM [16,17] (Figure 1). This interplay between kinases and phosphatases fine tunes the speed of the clock to $\sim 24 \mathrm{hr}$ by regulating the stability of PER and TIM and the timing of PER/TIM nuclear entry. PER phosphorylation sites targeted by PP2A and PP1 have been mapped in cell culture, but precise action of PP2A and PP1 on PER (and TIM) in vivo still needs to be elucidated [7]. In summary, a detailed picture is emerging of cascades of phosphorylation and dephosphorylation events that regulate PER and TIM stability, dimer formation and nuclear entry. These cascades introduce delays in the progression of the circadian cycle that are critical for period determination.

Regulation of PER and TIM stability is also important for communication between the small ventral lateral neurons (sLNvs) - the circadian pacemaker neurons - and the other circadian neurons of the fly brain [18]. The small LNvs secrete PIGMENT-DISPERSING FACTOR (PDF) to keep all circadian neurons properly synchronized $[19,20]$. The receptor for PDF (PDFR) is a G-protein coupled receptor (GPCR) that increases cAMP levels and thus PKA activity [21,22]. TIM appears to be a likely candidate target of PDFR signaling. Indeed, TIM harbors many putative PKA phosphorylation sites [23•] and can be phosphorylated by PKA in vitro [24]. When the sLNvs are forced to fire action potentials and thus secrete PDF early at night, this triggers TIM degradation in the dorsal lateral neurons (LNds), which is mediated by PDFR and CUL-3 [25•]. TIM was also found to be a primary target of PDF/ PDFR signaling in a separate study, but, paradoxically, constant reduction of PKA signaling resulted in lower TIM levels [23•]. Finally, PDFR signaling can also result in PKA-mediated PER stabilization [26]. These complex effects of PDFR/PKA signaling on critical circadian proteins could help explaining how PDF can either speed up or slow down circadian pacemakers in a neuron-specific manner [20]. It is also possible that acute PDF secretion triggers different molecular responses than long-term PDF signaling. Finally, DBT has recently been implicated in synchronization of PER and TIM oscillations in circadian neurons, possibly interacting with PDFR signaling [27]. It will be interesting to determine by which mechanism DBT contributes to neural synchronization.

\section{CLK phosphorylation and ubiquitination}

It has been suggested that the repression of the transcription factor complex CLK/CYC occurs after phosphorylation of CLK, which releases it from the DNA $[28,29]$. Similar to PER, CLK phosphorylation is also rhythmic with maximum phosphorylation state in the morning when CLK/CYC activity is lowest [28,30-32]. This phosphorylation is promoted by PER and DBT, but DBT's kinase activity is not required [33]. This suggests that PER and DBT are involved in the recruitment of other CLK kinases. CK2 $a$ and NEMO inhibit 
CLK/CYC (Figure 1). Indeed, phosphorylation by CK2a increases CLK stability but reduces its activity [34] and a dominant-negative form of CK2a, CK2a ${ }^{\text {Tik }}[14]$ results in increased CLK activity and decreased CLK levels. Complementing this effect, NEMO destabilizes CLK [35]. Indeed, NEMO downregulation using either RNAi or a hypomorphic allele results in higher CLK levels and short period. In addition, NEMO over-expression decreases CLK levels and consequently delays PER and TIM accumulation and causes long period. Recently, it was found that CLK is phosphorylated at $>15$ sites in cell culture [36,37]. Mutation of all of these sites to alanine (CLK-15A) results in hypo-phosphorylated CLK, which has increased stability and transcriptional activity. However, this mutant is still responsive to PER repression. As expected from these results, CLK-15A binds CYC and PER. It also rescues behavioral rhythmicity in a CLK null mutant, but with a short period [36]. per and tim mRNA cycles robustly in these rescued flies, indicating that the 15 phosphorylation sites identified in cell culture are not required for rhythmic PER repression of CLK activity in vivo.

Ubiquitination also plays a role in regulating CLK activity. CLK is ubiquitinated rhythmically with its peak at maximal transcriptional activity [38]. Interestingly, it is well established that ubiquitination promotes the activity of transcription factors, as well as their turnover [39]. UB-specific protease 8 (USP8) binds to CLK. USP8 downregulation or overexpression of a dominant negative mutant protein results in enhanced ubiquitination and transcriptional activity of CLK [38] (Figure 1). Since its mRNA is itself under circadian control [40-43], USP8 regulates its own transcription, as well as per, tim and that of other CCGs by inhibiting CLK transcriptional activity through deubiquitination. CLK stability is also regulated by the HECT-domain containing E3 UB-ligase CIRCADIAN-TRIP (CTRIP) [44] (Figure 1). CTRIP downregulation results in higher CLK levels and long period without affecting $C l k$ mRNA, which suggests that CLK stability is increased. CTRIP also destabilizes PER and TIM, independently of its effect on CLK (Figure 1). Taken together, CLK activity is tightly regulated by PER, CK2 $\alpha$ and USP8, and its stability controlled by NEMO and CTRIP. CLK repression is relieved after degradation of hyper-phosphorylated PER and TIM by SLIMB, thereby starting a new cycle.

\section{O-GIcNAcylation}

Recently, $O$-GlcNAcylation has been shown to regulate PER abundance and nuclear entry [45•]. This is a unique form of glycosylation in that it occurs both in the nucleus and cytoplasm and is reversible. $O$-GlcNAc transferase (OGT) enzyme deposits $\mathrm{N}-$ acetylglucosamine to its targets at Serine/Threonine residues, which can compete with phosphorylation. PER is a target of OGT and this modification competes with the maturation of PER by kinases [45] (Figure 1). PER's O-GlcNAcylation rhythms precede phosphorylation rhythms, but this does not appear to be driven by rhythms in OGT or $\beta-\mathrm{N}$ acetylglucosaminidase (OGA), the enzyme that removes $O$-GlcNAcylation. When OGT is overexpressed, PER nuclear entry is delayed and flies exhibit a long period phenotype. Moreover, OGT down-regulation results in the opposite phenotype: early nuclear entry of PER and short period. Additionally, PER stability was found to be increased by OGT, apparently through modulation of DBT-mediated PER degradation [45]. However, opposite effects on PER levels in vivo have recently been reported when manipulating $O$ - 
GlcNAcylation [46]. In this latter report, CLK was also identified as a target of OGT/OGA regulation. Since OGT activity is dependent on glucose levels, it was suggested that this modification may be an input mechanism from cellular metabolism to the circadian clock [46].

\section{Post-Transcriptional regulation mRNA stability}

While circadian transcriptional and post-translational regulations have been studied in detail, much less is known about the impact of RNA stability and translational control.

Interestingly however, an early study - based on run-on assays - had shown that stability of per mRNA changes during the course of the circadian cycle [47]. The recent dramatic advances in whole-genome expression analysis have led to the realization that mRNA stability control has a wide role in shaping circadian gene expression $[48 \bullet \bullet, 49]$. Indeed, analysis of nascent mRNAs in fly heads using high-throughput sequencing or nascent-seq has shown that about 130 nascent mRNAs show robust daily cycling [48••]. However, their mature mRNAs do not always follow this profile and can be either more or less robust in their cycling. Indeed, out of the 130 robustly cycling nascent mRNAs, only about a third of them resulted in rhythmic mature mRNA. Furthermore, only about $20 \%$ of the robustly rhythmic mRNA had cycling nascent RNAs. Similar results were shown in the mammalian system as well; with $70 \%$ of rhythmic mRNAs showing arrhythmic nascent RNA profile [49]. Thus, rhythmicity of an mRNA is not always tied to rhythmic transcriptional activity and post-transcriptional regulation plays a critical role in increasing or decreasing the robustness of mRNA rhythms.

\section{Translational regulation}

A novel high-throughput method that can detect mRNA-ribosome association specifically in circadian tissues of fly heads has demonstrated that about $10 \%$ of mRNAs show circadian changes in their translation [50•]. Furthermore, peak translation of these mRNAs occurs in two waves: at midday or mid-night when the flies are less active. Interestingly, mRNAs that are involved in similar pathways peaked at similar times, supporting functional relevance of time-specific translation. An interesting candidate for regulating rhythmically translation is the RNA binding protein LARK. This protein is required for normal eclosion and locomotor activity rhythms [51], and is expressed rhythmically with a peak late during the day [52]. A recent study has shown that LARK promotes DBT expression by binding to $d b t$ mRNAs, and indicates that this interaction controls circadian period length [53].

Proteins that are involved in the initiation of translation and RNA-binding proteins have a role in determining the robustness of per translation in Drosophila as well. Recently, it has been shown that TWENTY-FOUR (TYF) promotes per translation [54] (Figure 2). When TYF expression is reduced or lost, PER levels are decreased and as a result behavioral rhythms are weakened with a longer period. TYF does not carry any recognizable RNA binding domain, but can promote $l u c$ reporter gene translation when tethered to the luc mRNA through a heterologous RNA binding domain. Furthermore, TYF binds the $5^{\prime}$-cap binding complex and Poly-A Binding Protein (PABP), which might promote per mRNA 
circularization. Interestingly, TYF expression appears to be limited to pacemaker circadian neurons (the ventral and dorsal lateral neurons), and PER expression is only reduced in these neurons in tyf mutants [54]. ATAXIN-2 (ATX2), an RNA binding protein involved in several neuronal degenerative diseases in humans, forms a complex with TYF and is required for TYF's transactivation activity on per [55••,56••] (Figure 2). Through its ability to bind PABP, ATX2 promotes the formation of TYF/PABP complexes. ATX2 binds per mRNA, but is also able to bind other mRNAs. Thus, how TYF specifically binds to per mRNA remains unclear. In addition to TYF and ATX2, the atypical translation factor NAT1 - which is involved in cap-independent translation - also promotes per expression in circadian pacemaker neurons [57]. Why per translation is particularly tightly regulated in pacemaker neurons remains unknown. This level of control could be used to strengthen molecular rhythms in clock neurons, or serve as a point of entry for neural or environmental input pathways. Notably, exaggerated light responses were observed late at night when NAT1 was down-regulated [57], although this increased response could result from a weakened circadian oscillator [58].

miRNAs

Micro-RNAs (miRNAs) are short, single stranded non-coding RNAs that also regulate gene expression [59]. They repress translation and cause degradation of a wide variety of transcripts and have an impact on most aspects of biology. They would thus be anticipated to play an important role in the control of circadian rhythms. Supporting this idea, it was found that specific miRNAs are under circadian control and that some miRNAs are enriched in circadian tissues [60,61]. Moreover, co-immunoprecipitation of miRNAs with AGO1 antibodies revealed that $c l k$, vri and clockwork orange (cwo) are bound by miRNAs. One of these miRNAs, bantam, was found to bind $c l k$ at three binding sites in the $c l k$ 3'UTR [60] (Figure 2). Furthermore, overexpression of bantam leads to a long period phenotype, and clk 3'UTR that lacks bantam binding sites poorly rescues rhythmicity to the arrhythmic strain $c l k^{A R}$. This shows that regulation of $c l k$ mRNA by the bantam miRNA is required for robust rhythmicity in flies. In addition, it has been recently shown that CLK/CYC indirectly drives rhythmic expression of let-7 miRNA, which in turn negatively regulates CLOCKWORKORANGE (CWO), a rhythmically expressed transcriptional modulator of CLK/CYC transcription [62-66] (Figure 2). Over-expression of let-7 lengthens the circadian period, while its deletion causes partial arrhythmicity [62]. It appears that miRNAs are important for the control of circadian output pathways as well. Downregulation of GW182 - a protein that binds to AGO-1 and is essential for miRNA-dependent silencing of target genes - mimics the phenotype observed when PDF or its receptor are knocked-out [67•]. Indeed, GW182 genetically interact with PDFR signaling, and silences the expression of DUNCE (DNC), a cAMP phosphodiesterase, a negative regulator of cAMP levels and hence of PDFR signaling. Interestingly, this regulation is under light control and could help explain how light controls the respective impact of different circadian neurons on locomotor behavior. Indeed, PDF positive sLNvs have a more profound influence on locomotor behavior in constant darkness or under long nights, while the role of PDF negative neurons is promoted under constant light or under long day conditions [68-70]. Beside general downregulation of miRNA function, the role of specific miRNAs in output function has also been studied. Genomic deletions of miRNA-279 or its overexpression results in high level of behavioral 
arrhythmicity, but the core clock is still rhythmic and show normal PER oscillations in circadian neurons [71•]. miRNA-279 regulates unpaired (upd) in the JAK/STAT pathway. Consistent with this, up- or down-regulation of upd or JAK/STAT pathway also results in arrhythmicity, revealing the requirement of this pathway for normal circadian locomotor rhythms. In addition, the rhythmically expressed miR959-964 cluster was found to have an important impact on feeding time and immunity [72•]. Interestingly, the expression of this miRNA cluster is affected by food intake. Thus, a regulatory feedback loop influences circadian feeding through miRNA regulation. miR959-964 also have a weak effect on the period of circadian locomotor behavior, and might thus feedback on the molecular clock, at least in circadian neurons (Figure 2).

\section{Conclusion}

The study of the Drosophila circadian pacemaker first revealed the critical role of transcriptional rhythms in the generation of circadian rhythms, and allowed us to understand with remarkable details how rhythmic transcription is achieved. Our understanding of the mechanisms controlling protein stability (phosphorylation is particular) is rapidly catching up, and we begin to understand how cascades of phosphorylation and dephosphorylation can lead to the generation of stable 24-hr period rhythms. Moreover, intermediate regulatory mechanisms that affect mRNA metabolism and translation are emerging as critical for circadian rhythmicity. We are therefore on the brink of achieving a holistic view of the molecular mechanisms controlling circadian gene expression.

\section{Acknowledgement}

We apologize to researchers whose work was not discussed here due to space limitations. We thank Dr. Yong Zhang for carefully reading our manuscript. P. E. is supported by NIH R01 grants GM066777 and GM100091 from the National Institute of General Medical Sciences

\section{References}

1. Crane BR, Young MW. Interactive features of proteins composing eukaryotic circadian clocks. Annu Rev Biochem. 2014; 83:191-219. [PubMed: 24905781]

2. Reppert SM, Gegear RJ, Merlin C. Navigational mechanisms of migrating monarch butterflies. Trends Neurosci. 2010; 33:399-406. [PubMed: 20627420]

3. Tataroglu O, Emery P. Studying circadian rhythms in Drosophila melanogaster. Methods. 2014; 68:140-150. [PubMed: 24412370]

4. Hardin PE. Molecular genetic analysis of circadian timekeeping in Drosophila. Adv Genet. 2011; 74:141-173. [PubMed: 21924977]

5. Zhang, Y.; Emery, P. Molecular and neural control of insects circadian rhythms. In: Gilbert, LI., editor. Insect Molecular Biology and Biochemistry. New York: Academic Press; 2012. p. 513-551.Edited by: vol

6. Duvall LB, Taghert PH. Circadian rhythms: biological clocks work in phospho-time. Curr Biol. 2011; 21:R305-R307. [PubMed: 21549947]

7. Garbe DS, Fang Y, Zheng X, Sowcik M, Anjum R, Gygi SP, Sehgal A. Cooperative interaction between phosphorylation sites on PERIOD maintains circadian period in Drosophila. PLoS Genet. 2013; 9:e1003749. [PubMed: 24086144]

8. Ko HW, Jiang J, Edery I. Role for Slimb in the degradation of Drosophila Period protein phosphorylated by Doubletime. Nature. 2002; 420:673-678. [PubMed: 12442174] 
9. Grima B, Lamouroux A, Chelot E, Papin C, Limbourg-Bouchon B, Rouyer F. The F-box protein slimb controls the levels of clock proteins period and timeless. Nature. 2002; 420:178-182. [PubMed: 12432393]

10. Chiu JC, Vanselow JT, Kramer A, Edery I. The phospho-occupancy of an atypical SLIMB-binding site on PERIOD that is phosphorylated by DOUBLETIME controls the pace of the clock. Genes Dev. 2008; 22:1758-1772. [PubMed: 18593878]

11. Chiu JC, Ko HW, Edery I. NEMO/NLK phosphorylates PERIOD to initiate a time-delay phosphorylation circuit that sets circadian clock speed. Cell. 2011; 145:357-370. [PubMed: 21514639]

12. Lin JM, Kilman VL, Keegan K, Paddock B, Emery-Le M, Rosbash M, Allada R. A role for casein kinase 2alpha in the Drosophila circadian clock. Nature. 2002; 420:816-820. [PubMed: 12447397]

13. Martinek S, Inonog S, Manoukian AS, Young MW. A role for the segment polarity gene shaggy/ GSK-3 in the Drosophila circadian clock. Cell. 2001; 105:769-779. [PubMed: 11440719]

14. Meissner RA, Kilman VL, Lin JM, Allada R. TIMELESS is an important mediator of CK2 effects on circadian clock function in vivo. J Neurosci. 2008; 28:9732-9740. [PubMed: 18815259]

15. Grima B, Dognon A, Lamouroux A, Chelot E, Rouyer F. CULLIN-3 controls TIMELESS oscillations in the Drosophila circadian clock. PLoS Biol. 2012; 10:e1001367. [PubMed: 22879814] Identification of CULLIN-3 and SLIMB as key regulators of TIM stability, acting during two different phase of the circadian cycle.

16. Sathyanarayanan S, Zheng X, Xiao R, Sehgal A. Posttranslational regulation of Drosophila PERIOD protein by protein phosphatase 2A. Cell. 2004; 116:603-615. [PubMed: 14980226]

17. Fang Y, Sathyanarayanan S, Sehgal A. Post-translational regulation of the Drosophila circadian clock requires protein phosphatase 1 (PP1). Genes Dev. 2007; 21:1506-1518. [PubMed: 17575052]

18. Nitabach MN, Taghert PH. Organization of the Drosophila circadian control circuit. Curr Biol. 2008; 18:R84-R93. [PubMed: 18211849]

19. Lin Y, Stormo GD, Taghert PH. The neuropeptide pigment-dispersing factor coordinates pacemaker interactions in the Drosophila circadian system. J Neurosci. 2004; 24:7951-7957. [PubMed: 15356209]

20. Yoshii T, Wulbeck C, Sehadova H, Veleri S, Bichler D, Stanewsky R, Helfrich-Forster C. The neuropeptide pigment-dispersing factor adjusts period and phase of Drosophila's clock. J Neurosci. 2009; 29:2597-2610. [PubMed: 19244536]

21. Mertens I, Vandingenen A, Johnson EC, Shafer OT, Li W, Trigg JS, De Loof A, Schoofs L, Taghert PH. PDF receptor signaling in Drosophila contributes to both circadian and geotactic behaviors. Neuron. 2005; 48:213-219. [PubMed: 16242402]

22. Shafer OT, Kim DJ, Dunbar-Yaffe R, Nikolaev VO, Lohse MJ, Taghert PH. Widespread receptivity to neuropeptide PDF throughout the neuronal circadian clock network of Drosophila revealed by real-time cyclic AMP imaging. Neuron. 2008; 58:223-237. [PubMed: 18439407]

23. Seluzicki A, Flourakis M, Kula-Eversole E, Zhang L, Kilman V, Allada R. Dual PDF signaling pathways reset clocks via TIMELESS and acutely excite target neurons to control circadian behavior. PLoS Biol. 2014; 12:e1001810. [PubMed: 24643294] Identification of TIM as a critical downstream target of PDF-receptor/PKA signalling.

24. Zeng H, Qian Z, Myers MP, Rosbash M. A light-entrainment mechanism for the Drosophila circadian clock. Nature. 1996; 380:129-135. [PubMed: 8600384]

25. Guo F, Cerullo I, Chen X, Rosbash M. PDF neuron firing phase-shifts key circadian activity neurons in Drosophila. Elife. 2014; 3 Identification of TIM degradation by CUL-3 as a key mediator of PDF-receptor signalling

26. Li Y, Guo F, Shen J, Rosbash M. PDF and cAMP enhance PER stability in Drosophila clock neurons. Proc Natl Acad Sci U S A. 2014; 111:E1284-E1290. [PubMed: 24707054]

27. Zheng X, Sowcik M, Chen D, Sehgal A. Casein kinase 1 promotes synchrony of the circadian clock network. Mol Cell Biol. 2014; 34:2682-2694. [PubMed: 24820422] 
28. Yu W, Zheng H, Houl JH, Dauwalder B, Hardin PE. PER-dependent rhythms in CLK phosphorylation and E-box binding regulate circadian transcription. Genes Dev. 2006; 20:723733. [PubMed: 16543224]

29. Menet JS, Abruzzi KC, Desrochers J, Rodriguez J, Rosbash M. Dynamic PER repression mechanisms in the Drosophila circadian clock: from on-DNA to off-DNA. Genes Dev. 2010; 24:358-367. [PubMed: 20159956]

30. Lee C, Bae K, Edery I. The Drosophila CLOCK protein undergoes daily rhythms in abundance, phosphorylation, and interactions with the PER-TIM complex. Neuron. 1998; 21:857-867. [PubMed: 9808471]

31. Kim EY, Edery I. Balance between DBT/CKIepsilon kinase and protein phosphatase activities regulate phosphorylation and stability of Drosophila CLOCK protein. Proc Natl Acad Sci U S A. 2006; 103:6178-6183. [PubMed: 16603629]

32. Hung HC, Maurer C, Zorn D, Chang WL, Weber F. Sequential and compartment-specific phosphorylation controls the life cycle of the circadian CLOCK protein. J Biol Chem. 2009; 284:23734-23742. [PubMed: 19564332]

33. Yu W, Zheng H, Price JL, Hardin PE. DOUBLETIME plays a noncatalytic role to mediate CLOCK phosphorylation and repress CLOCK-dependent transcription within the Drosophila circadian clock. Mol Cell Biol. 2009; 29:1452-1458. [PubMed: 19139270]

34. Szabo A, Papin C, Zorn D, Ponien P, Weber F, Raabe T, Rouyer F. The CK2 kinase stabilizes CLOCK and represses its activity in the Drosophila circadian oscillator. PLoS Biol. 2013; 11:e1001645. [PubMed: 24013921]

35. Yu W, Houl JH, Hardin PE. NEMO kinase contributes to core period determination by slowing the pace of the Drosophila circadian oscillator. Curr Biol. 2011; 21:756-761. [PubMed: 21514156]

36. Lee E, Jeong EH, Jeong HJ, Yildirim E, Vanselow JT, Ng F, Liu Y, Mahesh G, Kramer A, Hardin $\mathrm{PE}$, et al. : Phosphorylation of a central clock transcription factor is required for thermal but not photic entrainment. PLoS Genet. 2014; 10:e1004545. [PubMed: 25121504]

37. Mahesh G, Jeong E, Ng FS, Liu Y, Gunawardhana K, Houl JH, Yildirim E, Amunugama R, Jones $\mathrm{R}$, Allen DL, et al. : Phosphorylation of the transcription activator CLOCK regulates progression through a approximately $24-\mathrm{h}$ feedback loop to influence the circadian period in Drosophila. J Biol Chem. 2014; 289:19681-19693. [PubMed: 24872414]

38. Luo W, Li Y, Tang CH, Abruzzi KC, Rodriguez J, Pescatore S, Rosbash M. CLOCK deubiquitylation by USP8 inhibits CLK/CYC transcription in Drosophila. Genes Dev. 2012; 26:2536-2549. [PubMed: 23154984]

39. Komander D, Rape M. The ubiquitin code. Annu Rev Biochem. 2012; 81:203-229. [PubMed: 22524316]

40. Claridge-Chang A, Wijnen H, Naef F, Boothroyd C, Rajewsky N, Young MW. Circadian regulation of gene expression systems in the Drosophila head. Neuron. 2001; 32:657-671. [PubMed: 11719206]

41. McDonald MJ, Rosbash M. Microarray analysis and organization of circadian gene expression in Drosophila. Cell. 2001; 107:567-578. [PubMed: 11733057]

42. Ueda HR, Matsumoto A, Kawamura M, Iino M, Tanimura T, Hashimoto S. Genome-wide transcriptional orchestration of circadian rhythms in Drosophila. J Biol Chem. 2002; 277:1404814052. [PubMed: 11854264]

43. Kula-Eversole E, Nagoshi E, Shang Y, Rodriguez J, Allada R, Rosbash M. Surprising gene expression patterns within and between PDF-containing circadian neurons in Drosophila. Proc Natl Acad Sci U S A. 2010; 107:13497-13502. [PubMed: 20624977]

44. Lamaze A, Lamouroux A, Vias C, Hung HC, Weber F, Rouyer F. The E3 ubiquitin ligase CTRIP controls CLOCK levels and PERIOD oscillations in Drosophila. EMBO Rep. 2011; 12:549-557. [PubMed: 21525955]

45. Kim EY, Jeong EH, Park S, Jeong HJ, Edery I, Cho JW. A role for O-GlcNAcylation in setting circadian clock speed. Genes Dev. 2012; 26:490-502. [PubMed: 22327476] Demonstration that OGlcNAcylation plays a critical role in the circadian clock, regulating PER maturation. 
46. Kaasik K, Kivimae S, Allen JJ, Chalkley RJ, Huang Y, Baer K, Kissel H, Burlingame AL, Shokat KM, Ptacek LJ, et al. : Glucose sensor O-GlcNAcylation coordinates with phosphorylation to regulate circadian clock. Cell Metab. 2013; 17:291-302. [PubMed: 23395175]

47. So WV, Rosbash M. Post-transcriptional regulation contributes to Drosophila clock gene mRNA cycling. EMBO J. 1997; 16:7146-7155. [PubMed: 9384591]

48. Rodriguez J, Tang CH, Khodor YL, Vodala S, Menet JS, Rosbash M. Nascent-Seq analysis of Drosophila cycling gene expression. Proc Natl Acad Sci U S A. 2013; 110:E275-E284. [PubMed: 23297234] Characterization of cycling nascent/mature RNAs reveals the important contribution of posttranscriptional mechanisms to the robustness of mRNA rhythms.

49. Menet JS, Rodriguez J, Abruzzi KC, Rosbash M. Nascent-Seq reveals novel features of mouse circadian transcriptional regulation. Elife. 2012; 1:e00011. [PubMed: 23150795]

50. Huang Y, Ainsley JA, Reijmers LG, Jackson FR. Translational profiling of clock cells reveals circadianly synchronized protein synthesis. PLoS Biol. 2013; 11:e1001703. [PubMed: 24348200] Demonstration of broad translational regulation of gene expression in circadian tissues, with waves of protein synthesis.

51. Sundram V, Ng FS, Roberts MA, Millan C, Ewer J, Jackson FR. Cellular requirements for LARK in the Drosophila circadian system. J Biol Rhythms. 2012; 27:183-195. [PubMed: 22653887]

52. McNeil GP, Zhang X, Genova G, Jackson FR. A molecular rhythm mediating circadian clock output in Drosophila. Neuron. 1998; 20:297-303. [PubMed: 9491990]

53. Huang Y, McNeil GP, Jackson FR. Translational regulation of the DOUBLETIME/CKIdelta/ epsilon kinase by LARK contributes to circadian period modulation. PLoS Genet. 2014; 10:e1004536. [PubMed: 25211129]

54. Lim C, Lee J, Choi C, Kilman VL, Kim J, Park SM, Jang SK, Allada R, Choe J. The novel gene twenty-four defines a critical translational step in the Drosophila clock. Nature. 2011; 470:399_ 403. [PubMed: 21331043]

55. Zhang Y, Ling J, Yuan C, Dubruille R, Emery P. A role for Drosophila ATX2 in activation of PER translation and circadian behavior. Science. 2013; 340:879-882. [PubMed: 23687048]

56. Lim C, Allada R. ATAXIN-2 activates PERIOD translation to sustain circadian rhythms in Drosophila. Science. 2013; 340:875-879. [PubMed: 23687047] Both reports identify ATX2 as a key regulator of circadian rhythms, working with TYF in pacemaker neurons to promote PER translation.

57. Bradley S, Narayanan S, Rosbash M. NAT1/DAP5/p97 and atypical translational control in the Drosophila Circadian Oscillator. Genetics. 2012; 192:943-957. [PubMed: 22904033]

58. Pittendrigh CS, Kyner WT, Takamura T. The amplitude of circadian oscillations: temperature dependence, latitudinal clines, and the photoperiodic time measurement. J Biol Rhythms. 1991; 6:299-313. [PubMed: 1773097]

59. Kim Y, Kim VN. MicroRNA factory: RISC assembly from precursor microRNAs. Mol Cell. 2012; 46:384-386. [PubMed: 22633486]

60. Kadener S, Menet JS, Sugino K, Horwich MD, Weissbein U, Nawathean P, Vagin VV, Zamore PD, Nelson SB, Rosbash M. A role for microRNAs in the Drosophila circadian clock. Genes Dev. 2009; 23:2179-2191. [PubMed: 19696147]

61. Yang M, Lee JE, Padgett RW, Edery I. Circadian regulation of a limited set of conserved microRNAs in Drosophila. BMC Genomics. 2008; 9:83. [PubMed: 18284684]

62. Chen W, Liu Z, Li T, Zhang R, Xue Y, Zhong Y, Bai W, Zhou D, Zhao Z. Regulation of Drosophila circadian rhythms by miRNA let 7 is mediated by a regulatory cycle. Nature Commun. 2014; 5:5549. [PubMed: 25417916]

63. Lim C, Chung BY, Pitman JL, McGill JJ, Pradhan S, Lee J, Keegan KP, Choe J, Allada R. Clockwork orange encodes a transcriptional repressor important for circadian-clock amplitude in Drosophila. Curr Biol. 2007; 7:1082-1089. [PubMed: 17555964]

64. Kadener S, Stoleru D, McDonald M, Nawathean P, Rosbash M. Clockwork Orange is a transcriptional repressor and a new Drosophila circadian pacemaker component. Genes Dev. 2007; 21:1675-1686. [PubMed: 17578907]

65. Matsumoto A, Ukai-Tadenuma M, Yamada RG, Houl J, Uno KD, Kasukawa T, Dauwalder B, Itoh TQ, Takahashi K, Ueda R, Hardin PE, Tanimura T, Ueda H. A functional genomics strategy 
reveals clockwork orange as a transcriptional regulator in the Drosophila circadian clock. Genes Dev. 2007; 21:1687-1700. [PubMed: 17578908]

66. Richier B, Michard-Vanhée C, Lamouroux A, Papin C, Rouyer F. The clockwork orange Drosophila protein functions as both an activator and a repressor of clock gene expression. J Biol Rhythms. 2008; 23:103-116. [PubMed: 18375860]

67. Zhang Y, Emery P. GW182 controls Drosophila circadian behavior and PDF-receptor signaling. Neuron. 2013; 78:152-165. [PubMed: 23583112] Demonstration of the critical role played by miRNA in modulation of PDF-receptor signalling through regulation of dunce expression

68. Murad A, Emery-Le M, Emery P. A subset of dorsal neurons modulates circadian behavior and light responses in Drosophila. Neuron. 2007; 53:689-701. [PubMed: 17329209]

69. Stoleru D, Nawathean P, Fernandez MP, Menet JS, Ceriani MF, Rosbash M. The Drosophila circadian network is a seasonal timer. Cell. 2007; 129:207-219. [PubMed: 17418796]

70. Picot M, Cusumano P, Klarsfeld A, Ueda R, Rouyer F. Light activates output from evening neurons and inhibits output from morning neurons in the Drosophila circadian clock. PLoS Biol. 2007; 5:e315. [PubMed: 18044989]

71. Luo W, Sehgal A. Regulation of circadian behavioral output via a MicroRNAJAK/ STAT circuit. Cell. 2012; 148:765-779. [PubMed: 22305007] Characterization of a novel locomotor output mechanism regulated by miRNA 279

72. Vodala S, Pescatore S, Rodriguez J, Buescher M, Chen YW, Weng R, Cohen SM, Rosbash M. The oscillating miRNA 959-964 cluster impacts Drosophila feeding time and other circadian outputs. Cell Metab. 2012; 16:601-612. [PubMed: 23122660] Characterization of the rhythmic expression of a cluster of miRNAs and their roles in circadian feeding and immune response 


\section{Highlights}

- $\quad$ The Drosophila circadian pacemaker consists of transcriptional feedback loops

- $\quad$ Cascades of protein phosphorylation/dephosphorylation determine period length

- $\quad$ Specific ubiquitin ligases and proteases regulate circadian protein stability and activity

- $\quad$ mRNA stability and translation control emerge as key circadian regulatory mechanisms 


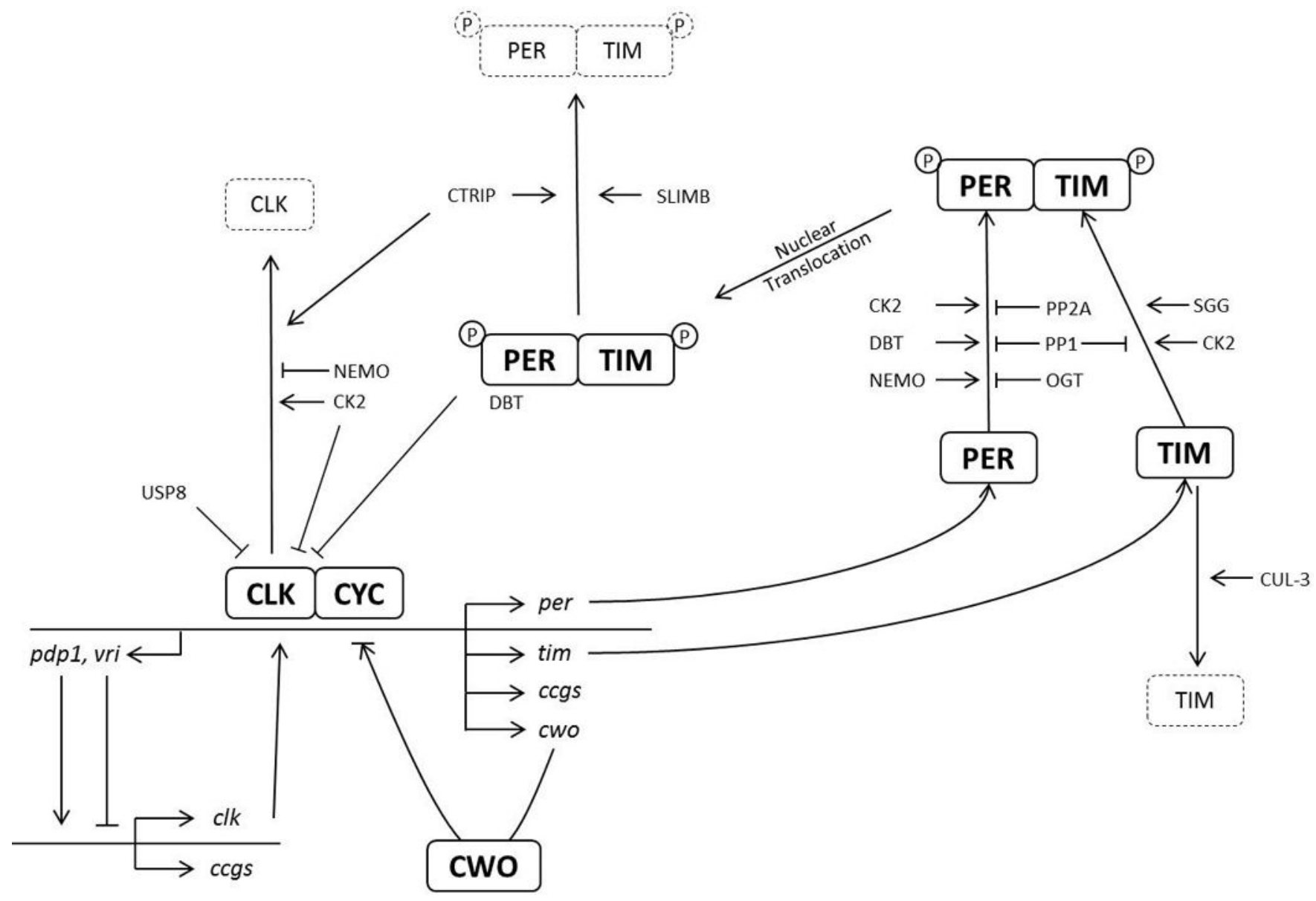

Figure 1.

Conceptual schematic of the Drosophila circadian molecular clock. Heterodimeric transcription factor complex CLK/CYC drives the expression of many clock-controlled genes (ccgs). Among them, per and tim encode critical transcriptional repressors of CLK/ CYC. This core feedback mechanism is tightly regulated at the transcriptional, posttranscriptional and posttranslational levels to introduce delays and checks to ensure a $\sim 24 \mathrm{hr}$ cycle. This figure emphasizes transcriptional and post-translational mechanisms. Arrows $(\rightarrow)$ indicate positive/enhanced regulation, while blunt-ends $(-t)$ indicate negative/ inhibitory regulation. CWO appears to be able to function either as a repressor or an activator [66]. Protein degradation is depicted with dashed lines. 


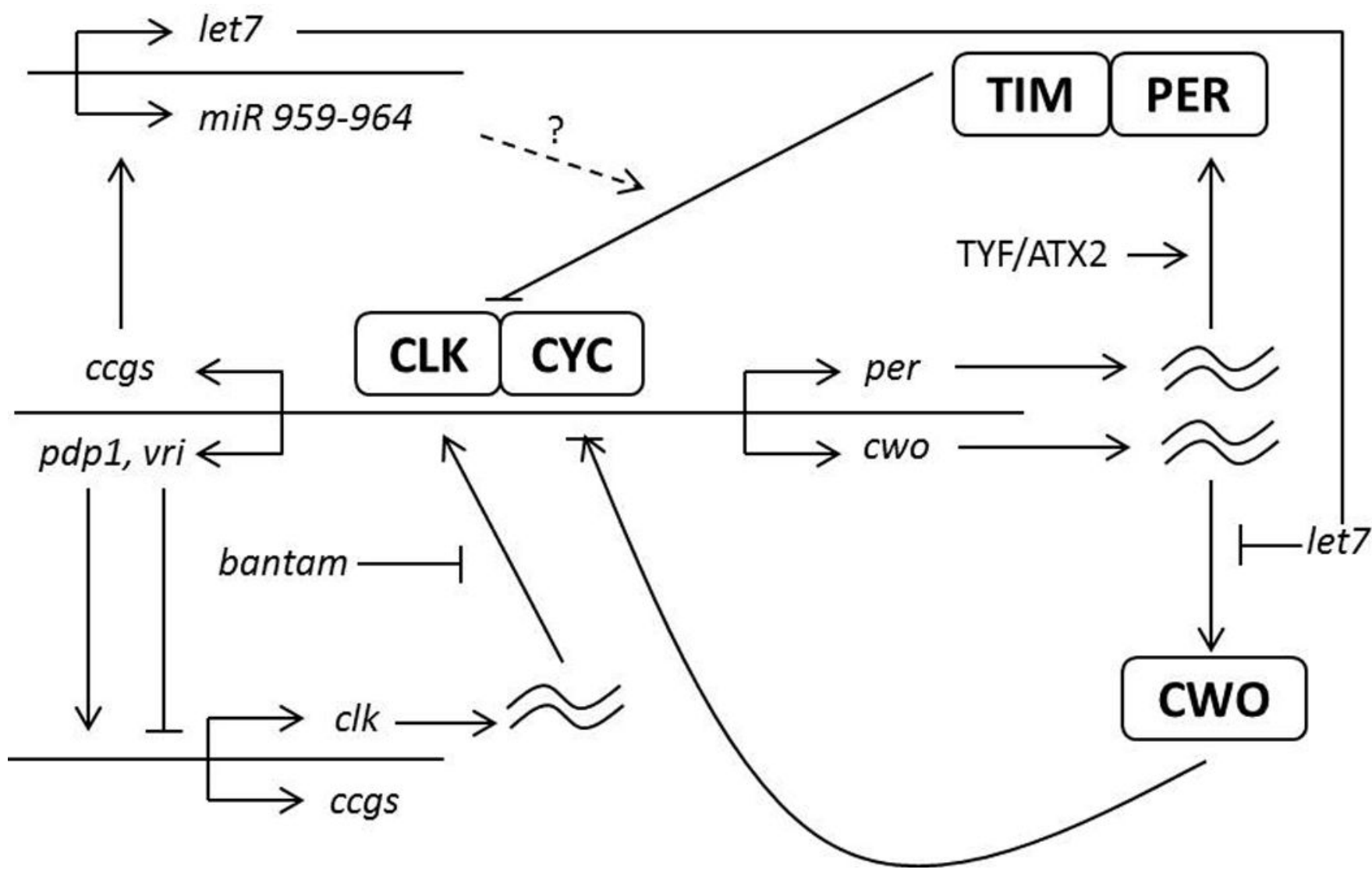

Figure 2.

Translational control mechanisms in the Drosophila circadian molecular clock. The microRNAs bantam and let7 regulate CLK and CWO expression, respectively. miR959964, which regulates feeding and immunity, also have a mild effect on the period of circadian behavior rhythms and thus the molecular clock of circadian neurons. The mechanism is not yet known (dotted line). This miRNA cluster and let 7 are under circadian control. ATX2/TYF promote specifically PER translation in circadian neurons by binding to per mRNA. 